\title{
LA RADIO COMO PROYECTO ESCOLAR DENTRO DE LA EDUCACIÓN INTERCULTURAL BILINGÜE EN ECUADOR
}

\author{
RADIO AS A SCHOOL PROJECT IN \\ INTERCULTURAL BILINGUAL \\ EDUCATION IN ECUADOR
}

doi: 10.21555/ rpp.v33i33.2418

Dayana Gabriela Aguirre Demera

dayagaby26@gmail.com

https: / / orcid.org/0000-0001-6875-5475
Diego Mauricio Suqui Pizarro

dpizarro1995@live.com

https:// orcid.org/0000-0002-3909-736X

Universidad Nacional de Educación, Ecuador

Recibido: octubre 17, 2021 - Aceptado: octubre 26, 2021

\section{RESUMEN}

La investigación parte de una propuesta pedagógica que emplea la radio como proyecto escolar en la Unidad Educativa del Milenio Intercultural Bilingüe Chibuleo, utilizando como metodología la investigación acción participativa, que permitió una relación horizontal y colaborativa entre todos los actores educativos. En las diferentes etapas se usaron instrumentos de investigación como el diario de campo, la observación participante, guías de análisis documental y las entrevistas; sin embargo, para el desarrollo de la propuesta, se empleó el Aprendizaje Basado en Proyectos (ABP), tal como lo propone el Manual para la elaboración de proyectos escolares en los centros educativos interculturales bilingües, dispuesto por la Secretaría de Educación Intercultural Bilingüe. Cada paso de esta metodología se adaptó a las distintas fases que constituyen la producción de un programa de radio y, a su vez, se adecuó al entorno virtual, construido con base en un trabajo colaborativo entre docente, alumno y comunidad, cumpliendo así con el objetivo de implementar una estrategia innovadora que fortaleciera las competencias digitales, el pensamiento crítico y la creatividad en los estudiantes.

A manera de conclusión puede afirmarse que la radio escolar es una estrategia pedagógica que mejora el aprendizaje interdisciplinar, fortaleciendo distintas habilidades y destrezas en el transcurso del proyecto -como su rol investigador, el trabajo colaborativo y su pertinencia cultural y lingüística-, contribuyendo en el desarrollo integral del estudiante y aportando significativamente al perfil de salida del estudiante de Educación Intercultural Bilingüe, por lo tanto, este proyecto es apto para replicar en otras instituciones educativas.

Palabras clave: aprendizaje basado en proyectos, interculturalidad, radio escolar, TIC trabajo colaborativo, virtualidad. 


\begin{abstract}
The research is based on a pedagogical proposal using radio as a school project in the Chibuleo Intercultural Bilingual Millennium Educational Unit, using participatory action research as a methodology that allowed a horizontal and collaborative relationship among all educational actors. In the different stages, research instruments such as the field diary, participant observation, documentary analysis guides and interviews were used; however, for the development of the proposal, Project Based Learning (PBL) was used, as proposed in the Manual for the elaboration of school projects in intercultural bilingual educational centers, provided by the Secretary of Intercultural Bilingual Education. Each step of this methodology has been adapted to the different phases that constitute the production of a radio program and in turn adapted to the virtual environment, built on the basis of collaborative work between teacher, student and community, thus fulfilling the objective of implementing an innovative strategy that strengthens digital skills, critical thinking and creativity in students.

In conclusion, it can be affirmed that school radio is a pedagogical strategy that improves interdisciplinary learning, strengthening different abilities and skills throughout the project, such as its investigative role, collaborative work and its cultural and linguistic relevance, contributing to integral development. of the student and significantly contributing to the exit profile of the student of Intercultural Bilingual Education, therefore, this project is suitable for replication in other educational institutions
\end{abstract}

Keywords: Project-based Learning, Interculturality, School Radio, ICT, Collaborative Work, Virtuality.

\title{
INTRODUCCIÓN
}

En el año 2020, la virtualidad pasó a ser el escenario principal en el ámbito educativo -por el brote del coronavirus COVID-19 a nivel mundial-, con el fin de resguardar la salud de todos los estudiantes, docentes y el personal administrativo de las distintas instituciones educativas. Ello ha de continuar vigente hasta que la situación de contagio y riesgo disminuya considerablemente en los próximos meses del periodo 2021-2022.

Por tal motivo, los docentes y estudiantes del Ecuador tratan de adaptarse a esta nueva modalidad de enseñanza-aprendizaje virtual; sin embargo, encuentran dificultades como la falta de conectividad, la carencia de dispositivos tecnológicos y el analfabetismo digital. Por consiguiente, el interés de esta investigación se centra en el apoyo de la radio escolar como herramienta didáctica innovadora que facilite el proceso educativo, en donde el estudiante asuma autonomía en su formación y el docente se convierta en un guía en este proceso.

La radio escolar brinda múltiples beneficios sustentados en una nueva didáctica. Figueroa (2006) señala: «las voces de los niños, niñas y jóvenes adolescentes que llenan los espacios del recreo con su alegría y calidez» crean un ambiente de socialización, de aprendizaje y colaboración, en el cual, pueden desarrollar su imaginación, destrezas y habilidades cognitivas. En este escenario, las competencias comunicativas, al igual que la participación, democracia, interculturalidad e interdisciplinariedad son elementos que deben fortalecerse.

Este proyecto propone utilizar la radio como una herramienta pedagógica, en la asignatura de Proyectos Escolares, como lo dictamina el Currículo Nacional Kichwa, según el Ministerio de Educación (Acuerdo Ministerial 440-13, p. 68), el cual responde a un espacio de aprendizaje interactivo, fortaleciendo el trabajo colaborativo interdisciplinario a través 
de un asunto de interés común, empleando el método del ABP. Desde la observación participante en la Unidad Educativa del Milenio Intercultural Bilingüe Chibuleo, y a través de la educación virtual, se buscó reforzar y evaluar los distintos conocimientos, las habilidades y destrezas en las clases virtuales, y en el hogar, a partir de distintas actividades expuestas en cada momento de la propuesta.

\section{DESARROLLO}

La radio constituye una herramienta didáctica que permite al estudiante construir sus propios conocimientos bajo una motivación intrínseca, fortaleciendo significativamente su rendimiento académico. La flexibilidad de esta herramienta permite enfocarse en la parte sonora y auditiva, sin requerir de imágenes visuales para su entendimiento. Por tal motivo, puede aplicarse desde los primeros años de educación infantil. Melgarejo y Rodríguez (2013) mencionan que los niños, al utilizar la radio en una etapa de 3 a 6 años de edad, pueden aprender a sentir, ver y oír con la ayuda de los símbolos, estimulando su mente y lenguaje.

En cambio, Padilla (2015) en su texto La radio escolar como estrategia didáctica integradora en el décimo grado de la institución educativa San Simón, establece distintas sesiones de trabajo para su implementación, pero en el desarrollo, los estudiantes demostraron desinterés, ya que lo identificaban como un espacio de entretenimiento más no de aprendizaje. Por lo cual, con distintas actividades, se logró consolidar un vínculo con la comunidad y la institución educativa, ya que a través de un diálogo profesor-estudiante, se fortalecieron las destrezas comunicativas, estimulando sus competencias y habilidades.

Caldera (2013) señala en su texto, La radio escolar: un medio para fortalecer la argumentación oral en los estudiantes del grado sexto de la Institución Educativa Simón Bolívar de Planeta Rica Córdoba, un proyecto organizado en parejas para desarrollar investigaciones previas sobre un tema en común; posteriormente se establecieron sesiones de ensayo para que los estudiantes, confiados de sí mismos, entraran al aire. Cada semana se evaluaban distintas destrezas: tono de voz, vocalización, coherencia en el discurso, argumentos claros y válidos..., lo que les permitía mejorar sus habilidades y llegar a la conclusión de que la radio puede ser un aliado en el proceso de enseñanza-aprendizaje.

En estas investigaciones, el empleo de la radio educativa tiene un objetivo en común: fortalecer distintas habilidades o destrezas en los estudiantes y, así, romper con la monotonía de un aprendizaje tradicional. Por ello, este trabajo se fundamenta a partir del paradigma constructivista que, de acuerdo con Pérez (2005), no solo facilita la interacción con el contexto u otros actores que observan, sino que es un proceso reflexivo y de autocrítica que resulta en nuevas perspectivas sobre lo observado. La razón de que esta investigación se sustente en el constructivismo se debe a que, durante el proceso investigativo, se mantuvo contacto y participación entre los sujetos de la población, surgiendo así una propuesta que responde a esta interacción conjunta que, de igual forma, se aplicó en su debido momento.

La propuesta fue desarrollada de manera virtual debido a la situación de riesgo que atravesaba el país. Espino y Martín (2012) afirman que el uso de la TIC optimiza los procesos de producción, comunicación, transmisión, difusión y recepción de contenidos radiofónicos a través de las diversas plataformas multimedia, lo que convierte a la radio en un medio híbrido. 


\section{Método de investigación}

Se fundamenta en un método de Investigación Acción Participativa (IAP), aludiendo a la participación activa que tienen los sujetos involucrados en los procesos investigativos del proyecto. Balcázar (2003), lo plantea desde un punto de vista epistemológico de aprender a aprender, rompiendo con los esquemas tradicionalista de la investigación. La IAP mejora los procesos investigativos de los estudiantes a partir de:

- Desarrollar sus habilidades de análisis desde una visión crítica que puedan aplicar, más adelante, en cualquier situación.

- Aprender a conducir el proceso de investigación: indagar información confiable en internet, comunicarse con grupos $\mathrm{u}$ organizaciones semejantes para adquirir su apoyo y mejorar recursos...

- Entender su rol en el proceso de transformación de la realidad social, como actores principales del cambio.

- Promover un desarrollo de conciencia crítica entre todos los participantes de la IAP.

\section{Enfoque de la investigación}

Esta investigación posee un enfoque inductivo asociado a la investigación cualitativa, planteando razonamientos ascendentes que circulan desde lo individual hasta lo general. $\mathrm{El}$ «método se observa, estudia y se conocen las características genéricas o comunes que se reflejan en un conjunto de realidades para elaborar una propuesta [...] de índole general» (Abreu, 2014, p. 6).

Se utilizó este método y enfoque debido a que cumple los intereses de la propuesta investigativa de la radio escolar, mediante la construcción y aplicación. De igual manera, sus características facilitaron mantener una interacción activa con los sujetos involucrados para, desde su percepción, cambiar las dificultades presentes en su entorno.

\section{Población y muestra}

En esta investigación, participaron 21 estudiantes de séptimo año de educación general básica paralelo «B», de la Unidad Educativa del Milenio Intercultural Bilingüe Chibuleo, registrando a diez estudiantes del sexo femenino y 11 estudiantes del sexo masculino, oriundos del pueblo Chibuleo de la nacionalidad Kichwa.

\section{Métodos empíricos: técnicas de investigación e instrumentos de investigación}

Las técnicas e instrumentos de investigación que se implementaron se mencionan a continuación: 
Tabla 1

Técnicas e instrumentos de investigación

\begin{tabular}{|c|c|c|c|c|}
\hline TÉCNICAS & INSTRUMENTOS & $\begin{array}{c}\text { OBJETIVOS DEL } \\
\text { TIC }\end{array}$ & $\begin{array}{l}\text { OBJETIVOS DEL } \\
\text { INSTRUMENTO }\end{array}$ & FUENTE \\
\hline Entrevista. & Guía de exploración. & $\begin{array}{l}\text { Identificar los } \\
\text { principales } \\
\text { saberes y } \\
\text { conocimientos } \\
\text { de la comunidad } \\
\text { Chibuleo, a } \\
\text { desarrollarse en } \\
\text { el programa de la } \\
\text { radio escolar. }\end{array}$ & $\begin{array}{l}\text { Recolectar } \\
\text { información sobre } \\
\text { los beneficios } \\
\text { del uso de la } \\
\text { radio como } \\
\text { una estrategia } \\
\text { didáctica en } \\
\text { la educación; } \\
\text { además, si de esta } \\
\text { manera se pueden } \\
\text { mantener y seguir } \\
\text { transmitiendo } \\
\text { los saberes y } \\
\text { conocimientos de } \\
\text { la comunidad. }\end{array}$ & $\begin{array}{l}\text { Elaboración } \\
\text { propia. }\end{array}$ \\
\hline $\begin{array}{l}\text { Análisis } \\
\text { documental. }\end{array}$ & $\begin{array}{l}\text { Guía de análisis } \\
\text { documental } \\
\text { curricular. }\end{array}$ & $\begin{array}{l}\text { Identificar } \\
\text { los saberes y } \\
\text { dominios de } \\
\text { la fase DDTE, } \\
\text { que pueden ser } \\
\text { desarrollados con } \\
\text { la integración de } \\
\text { saberes propios } \\
\text { de la comunidad, } \\
\text { durante el proceso } \\
\text { de enseñanza- } \\
\text { aprendizaje. }\end{array}$ & $\begin{array}{l}\text { Conocer } \\
\text { los saberes } \\
\text { curriculares } \\
\text { correspondientes } \\
\text { al proceso DDTE, } \\
\text { y vincularlos } \\
\text { con los saberes y } \\
\text { conocimientos de } \\
\text { la comunidad. }\end{array}$ & $\begin{array}{l}\text { Elaboración } \\
\text { propia. }\end{array}$ \\
\hline
\end{tabular}




\begin{tabular}{|c|c|c|c|c|}
\hline $\begin{array}{l}\text { Entrevistas semi } \\
\text { estructuradas. }\end{array}$ & Guía de entrevistas. & $\begin{array}{l}\text { Determinar la } \\
\text { metodología } \\
\text { para utilizar la } \\
\text { radio escolar } \\
\text { en los procesos } \\
\text { de enseñanza- } \\
\text { aprendizaje } \\
\text { interdisciplinares } \\
\text { integrados. }\end{array}$ & $\begin{array}{l}\text { El objetivo de } \\
\text { esta entrevista } \\
\text { tiene la finalidad } \\
\text { de recolectar } \\
\text { información sobre } \\
\text { los beneficios } \\
\text { del uso de la } \\
\text { radio como } \\
\text { una estrategia } \\
\text { didáctica en } \\
\text { la educación; } \\
\text { además, si de esta } \\
\text { manera se pueden } \\
\text { mantener y seguir } \\
\text { transmitiendo } \\
\text { los saberes y } \\
\text { conocimientos de } \\
\text { la comunidad. }\end{array}$ & $\begin{array}{l}\text { Elaboración } \\
\text { propia. }\end{array}$ \\
\hline $\begin{array}{l}\text { Análisis } \\
\text { documental. }\end{array}$ & $\begin{array}{l}\text { Guía de análisis } \\
\text { documental. }\end{array}$ & & $\begin{array}{l}\text { Conocer los } \\
\text { elementos que } \\
\text { integra un } \\
\text { programa de radio } \\
\text { y cómo elaborarlo. }\end{array}$ & \\
\hline $\begin{array}{l}\text { Observación } \\
\text { participante. }\end{array}$ & $\begin{array}{l}\text { - Guía de } \\
\text { observación. } \\
\text { - Diarios de campo. }\end{array}$ & $\begin{array}{l}\text { Diseñar los } \\
\text { programas } \\
\text { radiales, junto con } \\
\text { los estudiantes, } \\
\text { para fortalecer } \\
\text { los procesos } \\
\text { de enseñanza- } \\
\text { aprendizaje, } \\
\text { con pertinencia } \\
\text { cultural } \\
\text { lingüística. }\end{array}$ & $\begin{array}{l}\text { Realizar un } \\
\text { análisis del } \\
\text { proceso de } \\
\text { enseñanza- } \\
\text { aprendizaje } \\
\text { virtual y sus } \\
\text { dimensiones } \\
\text { (relacional y } \\
\text { pedagógica), } \\
\text { gestionadas en } \\
\text { el séptimo año } \\
\text { paralelo «B» de la } \\
\text { Unidad Educativa } \\
\text { del Milenio } \\
\text { Intercultural } \\
\text { Bilingüe Chibuleo. }\end{array}$ & $\begin{array}{l}\text { Elaboración } \\
\text { propia. }\end{array}$ \\
\hline
\end{tabular}

\section{PROPUESTA DEL APRENDIZAJE BASADO EN PROYECTOS}

La siguiente propuesta se orienta a partir de los distintos lineamientos pedagógicos que emergen mediante la observación de las prácticas pre profesionales y el análisis de las herramientas curriculares que otorga el Ministerio de Educación y la Subsecretaría de Educación 
Intercultural Bilingüe, como son, el Modelo del Sistema de Educación Intercultural Bilingüe (MOSEIB), el instructivo de proyectos escolares, los lineamientos pedagógicos para la implementación del MOSEIB 2017, el Currículo Nacional Kichwa y el libro Producción de programas de radio del autor Mario Kaplún, en colaboración con la CIESPAL.

\section{PRIMER MOMENTO}

Se aplicó una encuesta con dos preguntas principales: ¿qué nombre le pondrías al programa de radio? y ¿qué asignatura es tu favorita?, con el fin de consolidar y anticipar los dos primeros pasos de esta etapa de organización. La encuesta se elaboró en la plataforma de Google Forms, por su fácil uso y acceso, utilizada frecuentemente en las actividades de la unidad educativa y el Ministerio de Educación; esto permitió una comunicación asincrónica con los estudiantes y una rápida recogida de información, además de acortar distancias. Se adjunta el enlace: https:/ / docs.google.com/forms/d/e/1FAIpQLScKQVMEbxIaR5coAomjZP6K8L3NWA0ZRD5Ii4neH4hBJawdDA/ viewform? usp=sf link

\section{Punto de partida}

Se seleccionó una herramienta, en este caso la radio, que contribuyera a la resolución de los problemas identificados y que ayudara a los estudiantes a fortalecer: su pensamiento crítico, valores cómo la ética, la responsabilidad y el compromiso en actividades no solo académicas sino también socioculturales, sociolingüísticas, socioemocionales, etcétera.

A través de una exposición oral, se socializó a los estudiantes y a la docente del aula, de manera general, sobre el empleo de la radio como un instrumento de educación y cuáles serían los roles que jugaría cada uno en la construcción del programa de radio. De igual manera, se desarrolló una lluvia de ideas para conocer sus puntos de vista sobre cómo llevar a cabo la radio escolar y que esta fuera un éxito. Ellos mencionaron:

- Trabajar por grupos para no acumular las tareas en una sola persona.

- Preguntar y pedir ayuda a la comunidad sobre cómo podría hacerse.

- Trabajar en las horas sincrónicas de clase para optimizar el tiempo.

- Ver videos en internet para saber cómo hacer el programa.

- Buscar un medio para presentar el proyecto.

La presentación contenía el objetivo del programa y la importancia que tiene en la educación al momento de desarrollarlo, por ejemplo: adaptación del mensaje al medio; ser investigadores y poder contrastar la información; fortalecer la imaginación y creatividad; desarrollar la expresión escrita y oral; trabajar colaborativamente; debatir y argumentar. Se enfatizó la importancia de este proyecto al ejemplificar programas radiales implementados en distintas instituciones educativas, donde los estudiantes crean el desarrollo y la producción, mientras que el docente es un facilitador y guía para el desenvolvimiento del programa. Aquí el enlace de la presentación: https://view.genial.ly/603dc51933effb0dbd38f67b/ presentation-genially-sin-titulo 


\section{Formación de equipos}

Para este segundo apartado, es importante atender los roles de los actores educativos. El estudiante tendrá un papel protagónico activo y el docente una función de guía. Mientras que, para la conformación de los equipos, deben considerarse los intereses comunes, tomando en cuenta la realidad institucional y edad de los estudiantes. Además, se requiere fomentar la participación de todo el alumnado y aprovechar sus destrezas y habilidades.

Así pues, para la conformación de los equipos, primero se realizó un cuestionario con la pregunta de opción múltiple: ¿cuál es tu asignatura favorita?, buscando descubrir su inclinación hacia las distintas áreas del conocimiento: matemáticas, ciencias naturales, estudios sociales, educación cultural y artística, y kichwa, de las cuales se desprendió cada segmento del programa.

Una vez conocidos los resultados, se distribuyeron a los veintiún estudiantes, según su asignatura favorita, de manera que se sintieran motivados a trabajar en la construcción de cada segmento. En el caso de los alumnos que escogieron una sola asignatura, fueron designados a sus respectivos equipos sin problema; a quienes seleccionaron más de una asignatura, se les distribuyó de acuerdo con las vacantes de cada grupo. Además, se nombraron dos responsables para la bienvenida y despedida, considerando sus aptitudes, seleccionando a un niño y una niña, respetando el principio de alternabilidad de género.

En la radio escolar se encuentran distintos cargos: director, jefe de programación, conductores, locutores, productores, reporteros y disc jockey (DJ's). Por lo cual, en este proyecto se consideraron aspectos como: modalidad virtual de trabajo, año escolar (séptimo EGB) y edad de los estudiantes, escogiendo cuatro puestos para la estructura de la emisora escolar, tal como se presenta a continuación:

- Director: Este rol es asignado al docente, quien además de escoger los elementos pedagógicos a insertarse dentro de la propuesta, también orienta el proceso pedagógico.

- Productor: De igual manera, este cargo se le atribuye al docente, ya que implica la edición del programa.

- Guionistas: Con la finalidad de que los estudiantes desarrollaran competencias comunicativas, fueron ellos mismos quienes redactaron sus guiones, de acuerdo con la temática correspondiente, en conjunto con sus compañeros de segmento.

- Locutores: Esta función también se designó a los estudiantes, procurando que todos tuvieran su espacio para hablar.

\section{Definición del producto final}

Para que el proyecto avanzara y su calidad de trabajo fuera notable, se establecieron tiempos para cada actividad establecida en esta etapa. En cada clase sincrónica, los estudiantes iban concretando cada una de las actividades establecidas, mencionadas a continuación:

- Segmento: escogieron el nombre de su grupo que fue conocido e integrado dentro del programa. Mediante dos sesiones, en grupos reducidos en la plataforma Zoom, realizaron una lluvia de ideas con nombres tentativos o en algunos casos, palabras, para reorganizarlos y formar un título creativo y cautivador a los oídos de los radioescuchas (Tabla 2). 
- Temática: los temas de cada segmento se tomaron del currículo Kichwa de la unidad 54, concerniente al séptimo año del proceso DDTE, unidad que se estaba cursando durante la aplicación de este proyecto, con el fin de fortalecer los saberes y conocimientos adquiridos en las clases sincrónicas, paralelamente a cada una de las sesiones del proyecto.

En una sola sesión, se plantearon a cada grupo todos los temas de la unidad correspondientes a su respectiva asignatura; mediante una discusión y reflexión grupal, seleccionaron un tema para desarrollarlo en cada segmento.

\section{Tabla 2}

Saberes y conocimientos curriculares de cada segmento

\begin{tabular}{|l|l|l|}
\multicolumn{1}{|c|}{ ASIGNATURA } & \multicolumn{1}{|c|}{ CONTENIDO } & \multicolumn{1}{c|}{ SEGMENTO } \\
\hline Ciencias Exactas. & $\begin{array}{l}\text { Medidas de tiempo: siglo, década, } \\
\text { lustro. M.3.2.23 }\end{array}$ & Los matemáticos. \\
\hline Ciencias Naturales. & $\begin{array}{l}\text { Mamíferos: características internas } \\
\text { y externas. CN.3.1.5, CN.3.1.9, } \\
\text { CN.3.1.10 }\end{array}$ & La ciencia y la naturaleza. \\
\hline Ciencias Sociales. & $\begin{array}{l}\text { El levantamiento indígena de 1990. } \\
\text { CS.3.1.55, CS.3.1.62, CS.3.3.10, } \\
\text { CS.3.3.12 }\end{array}$ & $\begin{array}{l}\text { Aprendamos juntos sobre la } \\
\text { historia. }\end{array}$ \\
\hline Cultura Estética. & $\begin{array}{l}\text { Investigación de la artesanía: el } \\
\text { tejido. ECA.3.3.9 }\end{array}$ & Alegría y arte. \\
\hline $\begin{array}{l}\text { Lengua y Literatura } \\
\text {-Kichwa. }\end{array}$ & $\begin{array}{l}\text { Poemas y elementos literarios: } \\
\text { imágenes, comparación y } \\
\text { personificación. LL.3.1.2, LL3.3.10, } \\
\text { LL.3.3.7, LL.3.4.2, LL3.5.3 }\end{array}$ & $\begin{array}{l}\text { Aprendamos juntos y } \\
\text { sabemos más. }\end{array}$ \\
\hline
\end{tabular}

- Nombre del proyecto: Para establecerlo, se envió con anterioridad la encuesta antes mencionada con la pregunta: «¿qué nombre le pondrías al programa de radio?», para que los mismos estudiantes reflexionaran y consideraran un nombre apropiado. Sus respuestas fueron variadas, pero existían palabras repetidas entre todas las opciones, como: «Amigo - Aprendamos» por lo que se procedió a reorganizarlas y combinarlas, formando así un nombre tentativo para presentarlo en la siguiente sesión de trabajo. El nombre "Aprendamos entre amigos», fue aprobado por los 19 estudiantes presentes en la reunión sincrónica, como nombre oficial del programa de radio.

Para finalizar este apartado, se estableció un objetivo general y distintos objetivos específicos que permitieron establecer la dirección y meta del proyecto a alcanzar. Por ello, esta actividad se le atribuyó al docente, ya que era la persona que dirigía el proyecto y conocía los motivos para implementarlo. 
El objetivo general y los objetivos específicos de este proyecto de radio fueron:

- Objetivo general: producir el programa radial «Aprendamos entre amigos», con los estudiantes de séptimo año de educación general básica paralelo «B», para fortalecer las competencias digitales, el pensamiento crítico y la creatividad.

- Objetivos especificos:

- Seleccionar los grupos de trabajo para los distintos segmentos.

- Investigar y organizar la información, plasmándola en el guión del segmento correspondiente.

- Ejecutar la producción final del programa de radio.

- Ejecutar el programa de radio.

\section{SEGUNDO MOMENTO}

\section{Organización y planificación}

Para este punto de organización y planificación, se plantearon tiempos determinados en los cuales llevar a cabo las distintas actividades dispuestas en la etapa de investigación y producción final. A la vez, mediante un organizador gráfico en forma hexagonal, se concretó la integración y relación entre saberes y conocimientos de la comunidad, y los saberes curriculares a desarrollarse, como se indica a continuación.

\section{Figura 1}

Planificación y organización del $A B P$
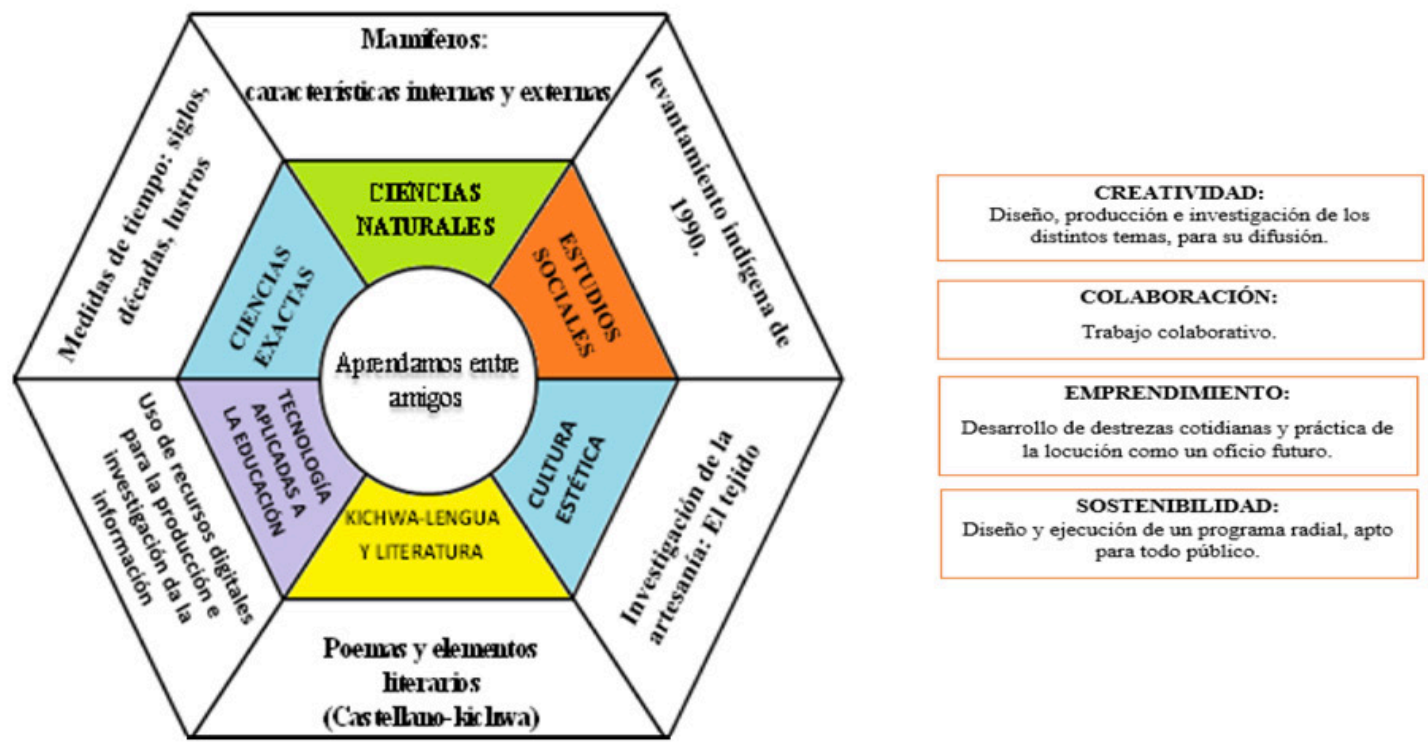

Nota. Adoptado de Manual para la elaboración de proyectos escolares en los centros educativos interculturales bilingües. 
El docente debe reflexionar y considerar los conocimientos del contexto -tanto de la unidad educativa como de la comunidad-, para realizar la organización y planificación. En este caso, el proyecto buscaba fortalecer los rasgos culturales y lingüísticos de la cultura Chibuleo.

De igual manera, para el programa de radio se adaptó una planificación específica, vinculada con el contexto áulico, misma que contenía los distintos elementos necesarios para la organización de un programa de radio: datos informativos; secciones; tiempos y estructura del guión, dispuestos dentro del esquema, de manera sistematizada y comprensible.

En cuanto al tiempo, se planteó que cada programa emitido tuviera una duración de diez minutos, tiempo adecuado para abarcar todos los temas de forma óptima y concreta, considerando que la edad de los estudiantes oscila entre 11 y 12 años, por lo que no se podía exigir un tiempo más prolongado. De igual manera, para cada sección se dispuso un límite de tiempo acorde a la extensión de información y dinámicas de las temáticas desarrolladas, con la finalidad de que los estudiantes incluyeran los datos que consideraran convenientes.

Por último, el docente organizó y dirigió los momentos sincrónicos o asincrónicos con los distintos grupos en torno a lo planificado, de manera que integraran las distintas actividades de forma paulatina hasta cumplir con todas las fases del programa.

\section{Búsqueda de información}

Para este apartado se centró la búsqueda, recopilación y comprensión individual de la información, basándose en conocimientos previos para, luego, incorporar nuevos conceptos y generar nuevos conocimientos. De esta manera, a los estudiantes, además de asumir el rol de locutores, también se les adjudicó el de investigadores encargados de buscar información para sus respectivos segmentos, con la intención de proponer contenido dentro del grupo de trabajo, impulsando así la construcción colectiva de saberes y conocimiento.

Para ello, se utilizó como recurso un taller sobre la búsqueda de información, dentro del cual se incluyeron definiciones de cada una de las fuentes y sus respectivos instrumentos para la recopilación de información, con el objetivo de que los estudiantes comprendieran las distintas fuentes de donde proviene la información y pudieran ampliar sus formas de investigación, y no solamente limitarse a la facilidad que ofrece la web.

Con el propósito de que los estudiantes identificaran los medios confiables, se les aconsejó cómo discriminar dichos medios para obtener información fidedigna. Finalmente, se presentaron cuatro pasos para la búsqueda de información: formular la necesidad de información; seleccionar fuentes de información apropiadas; preparar la búsqueda, y ejecutar y evaluar la información.

En el transcurso de este proceso, surgió la necesidad de encontrar una herramienta para organizar la información recogida por cada grupo, ya que varios estudiantes tenían problemas para recordar dónde anotaron lo que investigaron, debido a que la información estaba dispersa. En respuesta a este inconveniente, se construyó una ficha de investigación, trabajada de manera colaborativa en cada uno de los equipos: https://drive.google.com/drive/folders/ 1zpntbQLig4GRRrd1uHRYoV6HdECLs-5c?usp=sharing

Para este proceso, la guía del docente fue fundamental, pues era el responsable de dar seguimiento al trabajo realizado, dentro y fuera de las sesiones, según la organización de los estudiantes, ayudándoles a utilizar la ficha de forma correcta y a discernir la información recolectada. 
Además, se les sugirió incorporar los saberes culturales en cada segmento, con el objetivo de ir fortaleciendo la conexión con su pasado ancestral y, además, compartir esos conocimientos al mundo.

\section{Análisis y síntesis}

Los estudiantes realizaron un análisis exhaustivo y crítico de la información, para luego sintetizarla en cada uno de los diálogos. Es decir, seleccionaron los datos considerados importantes para incluir dentro del segmento y, posteriormente, redactaron su propio guión con ayuda de sus compañeros de grupo, apropiándose de los nuevos conocimientos y obteniendo una experiencia más significativa.

Previamente a la redacción del guión como tal, se realizó un esquema, organizando los aspectos a incluir en el segmento, de forma que quedara claro para el locutor dónde empezar; qué incluir después, cómo continuar con su tema y cuál sería la conclusión final. Este esquema también fue útil para determinar el tiempo de cada sección y la correcta distribución de los diálogos para cada estudiante.

En cuanto a la redacción, el primer punto a considerar fue el estilo ya que, al ser una emisora escolar dirigida a niños, era fundamental emplear un estilo no tan formal, comprensible y atractivo para el oyente. Otro punto a considerar fue el lenguaje, recordemos que el guión se escribió para transmitirse oralmente, por lo tanto, debía sonar con la sencillez, naturalidad y espontaneidad del lenguaje hablado. Una técnica útil fue el «escribir, escuchándose»: leer en voz alta cada diálogo mientras se escribía, o primero pronunciarlo para, luego, plasmarlo de manera escrita.

Finalmente, para obtener una redacción correcta se tomó en cuenta, por un lado, la sintaxis, es decir, se revisó si las ideas se presentaban de forma clara y, por otro lado, se examinó la puntuación, ya que ella indicaba al locutor dónde pausar. Para todo ello fue primordial la ayuda del profesor, quien está capacitado para detectar y corregir errores de esta índole.

\section{TERCER MOMENTO}

\section{Taller y producción}

En la última etapa del proyecto, los estudiantes practicaron los nuevos conocimientos adquiridos en su proceso de enseñanza-aprendizaje de las demás asignaturas, ejerciendo su rol de locutores dentro de la radio escolar. Para iniciar, se establecieron actividades que desarrollaran habilidades y destrezas de locución: dicción; hablar en público; mejorar sus expresiones orales y corporales; improvisar..., actividades que aplicaron en las distintas sesiones de trabajo:

- Juegos de roles: consiste en designar distintos personajes, relacionados a medios de comunicación, que los estudiantes pudieran interpretar (por ejemplo, conductores de televisión, locutores de noticias, presentadores deportivos, comentaristas de moda, etcétera). Era importante que el docente ejemplificara la actividad y dejara a los estudiantes a su libre albedrío sobre cómo interpretarla.

- El juego de las emociones: esta actividad radica en establecer un diálogo cualquiera con otra persona. Al momento de hablar, debían enfatizar una emoción (tristeza, alegría, risa, 
enojo, susto, sorpresa, etcétera). Esto permitía al estudiante manifestar sus emociones mediante la oralidad, la gesticulación y el perder el miedo al expresarse ante los demás.

- El helicóptero: la actividad permite trabajar los músculos faciales, por lo que se recomendó practicar, al menos, cinco minutos al día. Esto ayudaba a proyectar mejor la voz y volverla más nítida. El ejercicio reside en usar los labios, llenar de aire la cavidad bucal, y realizar un sonido de vibración, sintiéndolo a través de las mejillas y el cráneo. Ver el siguiente video: https:/ / www.youtube.com/watch?v=JE3Yn4-6znE

- El lápiz: se coloca un lápiz hexagonal en la boca y se le sujeta suavemente con los dientes, primero con los frontales, después con los caninos y, por último, con los premolares. Cuando ya se está sosteniendo, la persona debe tratar de leer y pronunciar claramente las palabras de una corta lectura; la dificultad aumenta cuando el lápiz se cambia de posición en los dientes. Ver el siguiente video: https: / www.youtube.com/watch?v=oy49t4XCqlo

- La respiración: se realizan ejercicios de respiración parecidos al yoga; por ejemplo: inhalar por la nariz y llenar de oxígeno el estómago (ampliando el diafragma) para, posteriormente, exhalar por la boca mientras se menciona la primera vocal del abecedario. Esta actividad permite entrenar la respiración para una lectura más fluida e ir controlando el oxígeno mientras se habla.

Es crucial que los estudiantes también comprendan la importancia del proyecto que realizan, ya que no solo consiste en trabajar hablando en un micrófono; su relevancia reside en exponer la información en una producción de calidad. Para ello, se establecieron dos conversatorios, impartidos por profesionales en el campo de la radio.

En el primer conversatorio, la doctora Daysi Salto, comunicadora social, con una experiencia en su campo a lo largo de treinta años, y diez años como docente, dio a conocer el valor de la radio en el medio social y el proceso de trabajo radial; abordó temas como la creación de una radio FM, equipo tecnológico, personal técnico, intereses, objetivos, importancia del locutor para emitir información, destrezas y habilidades que debe ir aprendiendo, distribución de tiempo, trato con los invitados y cuidado de la audiencia...

En cambio, en el segundo conversatorio, Joe Solano, estudiante investigador y miembro del colectivo «La estudiosa», compartió los conocimientos en la creación y producción de audios, proporcionando consejos y sugerencias de cómo lograr una calidad de sonido óptima en cada grabación, la repetición al frente de un espejo, el escucharse una y otra vez, y el correcto empleo de la grabadora de voz.

\section{Producto final de la propuesta}

Hasta esta parte del proyecto, los estudiantes ya habían logrado adquirir los conocimientos y las competencias necesarias para iniciar con el proceso de grabación de los audios que correspondían a cada segmento. Tomaron en cuenta los consejos recibidos por los profesionales en cuanto a vocalización, respiración y calidad de audio, para luego grabar sus respectivos diálogos, utilizando las distintas aplicaciones móviles sugeridas.

Para mayor comodidad, realizaron esta actividad de forma individual, a manera de tarea en casa, con la finalidad de recibir ayuda de sus representantes o de algún miembro de la familia, para luego ser enviada al docente a través de los distintos medios de comunicación como: WhatsApp, correo electrónico o Messenger. 


\section{Presentación del proyecto}

Una vez recopilados todos los audios, empezó el proceso de edición, actividad encargada al docente, como preparación para el siguiente paso. Las grabaciones realizadas fueron editadas mediante el programa «Adobe audition 3.0», software de fácil instalación y de uso simple.

Una vez agrupadas y editadas en un solo audio todas las grabaciones de los distintos segmentos, se presentó el resultado final a los estudiantes para que reflexionaran, reaccionaran y aprobaran el programa piloto, como producto del proyecto, a través de distintos medios como: YouTube, Facebook live y bocinas comunales.

\section{EVALUACIÓN DEL PROYECTO}

\section{Autoevaluación}

Este proceso permitió a los estudiantes identificar sus logros, dificultades, niveles de participación y cumplimiento de las actividades planificadas dentro del proyecto escolar. Para el diseño de los instrumentos se tomó en cuenta el desarrollo de las habilidades cognitivas, con base en la Taxonomía de Bloom, y las habilidades socioemocionales.

Se diseñó un cuestionario con seis preguntas abiertas, utilizando la herramienta de Google forms. Este fue aplicado al finalizar el proyecto, con la intención de que los estudiantes reflexionaran sobre su experiencia, desenvolvimiento y nuevos aprendizajes adquiridos a lo largo de todo el proceso, reconociendo sus fortalezas y debilidades por mejorar. https:// docs.google.com/forms/d/e/1FAIpQLSeBivMz]lRI5h7gIKuIRtICkzbyL60gcHnYb49cl0AQlbK1pQ/viewform

\section{Coevaluación}

Este tipo de evaluación se utilizó para fortalecer el trabajo colaborativo, determinando su importancia para los estudiantes, y promoviendo la opinión constructiva hacia los compañeros. Esta evaluación usó, como instrumento, una lista de cotejo virtualizada, a través de la plataforma Google forms, debido a su facilidad de empleo y adaptabilidad, con el objetivo de medir la participación de los compañeros de grupo, durante el trabajo colaborativo realizado en el proyecto escolar. https:/ / docs.google.com/forms/d/e/1FAIpQLSfCB4rlK6P7R7Ztq1fVl-wzypbRpgUM9yhkL53n-RFNiOSBaw/viewform?usp=sf_link

\section{Heteroevaluación}

Para esta evaluación se aplicó la técnica denominada «Felicito, critico, propongo», ya que permite evaluar la satisfacción de los participantes, utilizando sus resultados para mejorar el proceso de transformación del proyecto al recolectar las felicitaciones, críticas y propuestas para conseguir dicha mejora.

- Yo critico: mediante este apartado, se evidencian los valores que posee el grupo, así como también sus exigencias. 
- Yo felicito: permite resaltar los aspectos positivos, reconociendo el trabajo de todos los integrantes.

- Yo propongo: abre un espacio a la opinión de los participantes sobre cómo mejorar el proyecto y aplicar las sugerencias en un nuevo proceso.

En cuanto al facilitador, su función fue proporcionar un refuerzo positivo hacia las opiniones expuestas durante toda la actividad, enfatizando especialmente el análisis de las críticas, las cuales consideran aspectos susceptibles de mejora o aspectos negativos.

Esta actividad consistió en colocar, dentro de una matriz, los tres aspectos antes mencionados: felicito, critico, propongo. A continuación, se solicitó a los integrantes escribir una felicitación, una crítica y una propuesta sobre el proyecto. Una vez terminada esta tarea, cada estudiante colocaba su respuesta en la columna correspondiente. Acto seguido se leyeron las opiniones de todos, discutiendo las cuestiones que fueron surgiendo. Para finalizar se realizó una breve conclusión y se redactaron los resultados. (En el caso de que esta actividad se realice de forma individual, debe hacerse llegar a cada estudiante una hoja de trabajo con la matriz, de forma que pueda llenarla con las respuestas que considere oportunas).

Para la aplicación de esta técnica se empleó la plataforma Padlet, la cual funciona como una pizarra virtual, fácil de usar, que permite crear murales colaborativos. En cuanto su ejecución, se efectuó durante la última sesión de trabajo, en donde, con ayuda del docente, los estudiantes colocaron sus ideas debajo de cada apartado, procurando que todos los estudiantes participaran. https:/ / es.padlet.com/dayagaby26/f0ba6uwt6xm3t7iv

\section{Análisis de los resultados}

La propuesta fue una construcción continua a lo largo del año lectivo 2020-2021. Los datos se obtuvieron de los instrumentos de investigación como: diarios de campo y guía de observación; guía de exploración; las dos entrevistas semi estructuradas se triangularon en el software para análisis de datos cualitativos, gestión y creación de modelos Atlas T.I., a partir de distintas categorías, mostrando los principales momentos y las dinámicas del proceso de construcción de la propuesta pedagógica. A continuación, se presentan los principales datos obtenidos:

\section{- Competencias digitales}

Las competencias digitales fueron claves para desarrollar las distintas actividades académicas, investigativas y comunicativas dentro de todo el proceso de esta investigación acción participativa. Al estar sumergidos en una era tecnológica, es común que los estudiantes posean conocimientos en su uso, facilitando que puedan indagar, verificar o reforzar informaciones o nuevos conocimientos aprendidos en clase.

Como se señala en el diario de campo: «Los dispositivos electrónicos se convirtieron en un material esencial tanto para las clases sincrónicas, como para la entrega de tareas y práctica de la lectura». Estas competencias pueden fortalecerse en un contexto de educación virtual o presencial, todo depende de cómo el profesor encamina o planifica las distintas estrategias didácticas. A su vez, para implementarlas, debe reflexionar sobre el entorno del estudiante; es decir, si posee en su hogar las herramientas tecnológicas adecuadas para llevar a cabo la actividad (como redes wifi o dispositivos tecnológicos). 
Las competencias de comunicación o entornos digitales se reflejaron en el uso de aplicaciones como WhatsApp, Facebook, mensajes de texto, correos electrónicos, Work, Microsoft teams..., para llevar a cabo un seguimiento del desarrollo de la búsqueda de información y redacción de los guiones del programa de radio. Al utilizar estas aplicaciones diariamente en ámbitos sociales o académicos, los estudiantes no tuvieron problemas para emplearlas, pero sí se les dificultó el trabajo en equipo, porque algunos de sus compañeros de grupo se demoraban en responder, pero gracias a su perseverancia y responsabilidad en cumplir con las tareas, pudieron superar esa barrera.

Por otro lado, la resolución de problemas y creación de contenidos, como parte de la competencia digital, se plantearon en las primeras sesiones de trabajo del proyecto, al implementar nuevas aplicaciones o sistemas tecnológicos como Zoom, grabadoras de audio, Google drive o Google forms. Al ser desconocidas o utilizadas por primera vez estas herramientas, a los estudiantes se les dificultaba el manejo, por lo que se hicieron conversatorios y prácticas previas para solventar estos inconvenientes. Como mencionó un miembro de la comunidad: «El problema es cuando [...] el maestro no lo fortalece; algunos a veces solo esperan que llegue el sueldito y punto». Así pues, se decidió seguir reforzando las habilidades o destrezas que los estudiantes aun no dominaban, para evitar la idea de que el maestro no presta interés al éxito escolar del niño.

\section{- Habilidades cognitivas y socioemocionales}

La habilidad más significativa desarrollada en este proyecto fue la de ser investigador. Esto lo afirmaba un locutor de la comunidad: «Es necesario saber de todo un poco», es decir, si no se conoce un tema, debe indagarse y aprender sobre él, para que todo lo mencionado en el programa de radio sea verídico. De la misma manera, se enseñó al estudiante que siempre es importante exponer la verdad y verificar la información.

Otra de las habilidades que se fortaleció desde el inicio del proyecto, fue el liderazgo. Esto se logró conjuntamente con el trabajo colaborativo, ya que se requería de una buena organización entre los miembros del equipo; por ello, independientemente de los roles asignados dentro del proyecto, un integrante de cada grupo asumió el rol de líder, guiando, organizando y motivando al resto de sus compañeros en cada actividad, como lo indica los diarios de campo: «En cada equipo o grupo ya sea de trabajo, amigos o de estudios, hay una persona que resalta y ejerce el papel de líder, en este caso no hay excepción».

Esa fue una iniciativa propia que tuvieron los estudiantes, porque se dieron cuenta de que necesitaban una persona que los guiara y representara ante los demás. Los líderes de cada equipo fueron de gran ayuda al momento de realizar tareas que demandaban la tutela del docente, ya que, al ser cinco grupos, la revisión de las mismas se tornó compleja y el que existiera un estudiante a cargo, facilitó esta labor.

Cabe mencionar que, durante las clases, los estudiantes no participaban activamente con sus inquietudes o dudas, puesto que la única interacción existente se realizaba durante las clases sincrónicas, mediante el chat de la plataforma Teams. Por esta razón, se les complicó expresar sus opiniones durante las dos primeras sesiones de trabajo: tal vez porque sentían vergüenza o timidez hacia sus compañeros de clase o hacia los maestros, pero gracias a la insistencia de sus compañeros, comenzaron a manifestarse cada vez más, aunque no en su totalidad; por esta razón, la líder del grupo de ECA aconsejó a sus compañeros de los otros equipos que contribuyeran con información y que no se quedaran callados. 


\section{- Creatividad}

Se puede fortalecer a través de distintas actividades empleadas en el proceso de enseñanza-aprendizaje. Como afirmó la docente del aula: «Siempre es importante pensar qué actividades les pueden gustar a los niños, para que no se frustren o se aburran cuando lo realicemos en clase». Con esto pueden potenciarse habilidades cognitivas y socioemocionales, a fin de generar un aprendizaje significativo y eficaz.

El desarrollo del programa de radio requirió implementar actividades que fortalecieran la creatividad. Como mencionó la docente del aula: «[Necesitábamos] Un estudiante, cumpliendo con los roles de un locutor como: hablar en el micrófono, hacer entrevistas, poner música [...]». Son habilidades que debían destacar ante los demás, por ello era importante trabajarlas desde el inicio del proyecto, mediante ejercicios de dramatización relacionados al tema como: «comentaristas de noticias, comentarista deportivo, comentarista de moda, entrevistador de un programa, presentador del clima, entre otros personajes». Al realizar los ejercicios iniciales con los primeros estudiantes, todos sonrieron; esto fue el detonante para una participación activa y continua en las distintas dramatizaciones, porque querían demostrar su talento ante los demás, provocando una emoción para volver a participar, debido a que consideraban que, con la práctica, podrían mejorar, reflejando más emoción y carisma en cada intervención. Un estudiante explicó, por ejemplo: «Profe, ¿puedo volver a participar como presentador de noticias? Siento que puedo hacerlo mejor que mi compañero».

\section{- Pertinencia cultural y lingüística}

Este fue uno de los aspectos más importantes que se desarrolló en la investigación acción participativa, pues los estudiantes se comprometían a explorar y fortalecer sus lazos con los conocimientos de sus antepasados, mediante la difusión de su segmento. Como lo mencionó un locutor de la comunidad: «Pienso que aquí, también, los medios de comunicación tienen que hacer los programas culturales, económicos y sociales, porque es la base fundamental y también cultural», esto referido a que las emisoras de radio de la ciudad no fomentan la interculturalidad entre los espectadores. De ahí la importancia de crear una emisora o un programa de radio -ya sea con frecuencia periódica u online-que resuelva este dilema.

La pertinencia cultural y lingüística se fue fortaleciendo a través de la educación, con simples actividades que el estudiante pudo realizar, tanto en clase como en el hogar. Así lo mencionó la docente del aula: «Naturalmente busco actividades que se desarrollaban antiguamente en la comunidad, como es el tejido o la gastronomía, para [...] así también revitalizar los saberes ancestrales de la cultura kichwa o, en este caso, del pueblo Chibuleo».

Esto demuestra que no se necesitan grandes planificaciones o estrategias didácticas complicadas, ni para el docente ni para el estudiante, sino de una simple actividad -un canto, un poema, un tejido o elaborar alguna artesanía-, para fortalecer a gran escala la pertinencia cultural y lingüística. Solo depende de cómo el docente encamine la actividad y conozca el propósito al emplearla.

\section{- Dificultades de los docentes}

Uno de los inconvenientes que se presentaron en el transcurso del proyecto, fue la poca participación de los estudiantes en las distintas actividades grupales, por ejemplo, en el caso de la búsqueda de información y redacción de los guiones. La falta de comunicación interna en los distintos grupos, provocaba malestar entre los compañeros; a pesar de trabajar aquellas actividades en las distintas sesiones sincrónicas, necesitaban continuar en 
horas extracurriculares: «Ofrecimos nuestra ayuda, por si necesitaban trabajar por las tardes; de esto surgieron más inconformidades, en cuanto a los compañeros de grupo que no se conectaban». Lo que demuestra que el docente debe ser consciente en la distribución de tiempos para cualquier actividad y establecer un horario adecuado para evitar malestares entre compañeros de clase; debe tener en cuenta, también, un trabajo extra, fuera de su horario habitual de labores, para cumplir con sus responsabilidades profesionales.

Una de las dificultades de este proyecto, para el docente, era la de cumplir con su trabajo de edición del programa de radio, a partir de los audios que enviaban los estudiantes de sus respectivos segmentos. Sin embargo, algunos no enviaron el trabajo completo, a pesar de la insistencia por parte de sus compañeros de equipo y la del docente; esto provocó que el resultado final cambiara completamente y, de igual manera, el sentido del programa mismo. A ello se sumó lo complicado de la edición del audio, para lograr un programa entendible a los oídos de los radioescuchas.

\section{- Dificultades de los estudiantes}

Durante el transcurso del proyecto, los problemas en torno a la conectividad fueron un gran inconveniente para los estudiantes: la lejanía de su comunidad impedía una buena conexión por parte de los servicios de internet, que estaban en el centro de la ciudad; además, muchos no podían ingresar a las sesiones, porque no se les abría la aplicación o por los problemas de conexión wifi, o porque digitaron mal el código de acceso de la plataforma de reuniones de Zoom... Estos problemas se evidenciaron en la comunicación de algunos estudiantes, generando inconformidad para el resto de integrantes del grupo; de hecho, un estudiante solicitó cambiarse de equipo, debido a que los integrantes de su grupo (Ciencias Naturales) no contestaban a sus llamadas o mensajes. Por ello, es importante conocer la realidad de cada estudiante para comprender sus atrasos o problemas, y así buscar soluciones y evitar los malos entendidos.

\section{CONCLUSIONES}

La presente investigación evidencia que el uso de un programa radial, como herramienta didáctica, permitió a los estudiantes ejercer el rol protagónico en su proceso de enseñanza-aprendizaje; su autoestima y confianza crecieron en el transcurso del proyecto, mediante la capacidad de analizar, observar, reflexionar y calificar los sucesos de su entorno. A partir de las distintas actividades que se presentaron en la propuesta, se fortalecieron aquellas habilidades investigativas, creativas y de dicción, esenciales para un buen locutor y un estudiante sobresaliente.

De igual manera, los saberes y conocimientos del pueblo Chibuleo, fueron parte esencial de este proyecto que fortaleció la pertinencia cultural y lingüística de los estudiantes, al tener que indagar y verificar si la información que poseían sobre su cultura era correcta o si existía más información que debieran aprender para, así, transmitirla en su segmento del programa de radio. Todo ello con el fin de revitalizar e integrar los saberes ancestrales de su nacionalidad, en su proceso de enseñanza-aprendizaje, como se estipula en los armonizadores de saberes del MOSEIB.

Del mismo modo, se incluyeron las distintas áreas del conocimiento en un solo proyecto, facilitando el refuerzo académico de los saberes curriculares de la fase DDTE, aprendidos en las clases sincrónicas y, al mismo tiempo, desarrolladas con los saberes ancestrales de la comunidad. La educación de la EIB no solo se centra en la adquisición de los conocimientos 
científicos, sino también en la adquisición y práctica de los saberes y conocimientos de la nacionalidad.

Mediante este proyecto, los estudiantes crearon un programa de la radio escolar, plasmando nuevas ideas en cada actividad que iban realizando, en el transcurso de las tres etapas integradas del ABP. Estas actividades se planificaban conforme a las necesidades de los estudiantes, después de cada sesión de trabajo -ya fuera de lectura, creatividad e investigación-, cuyo fin era ir resolviendo las dificultades, dudas e inquietudes que se presentaban a lo largo del proyecto, bien fuera por sí mismos o con la ayuda de los miembros de su equipo, cumpliendo con éxito los ideales de un paradigma constructivista.

Para concluir, este diseño del programa de radio ayudó a fortalecer las competencias digitales, la pertinencia cultural y lingüística, el pensamiento crítico y la creatividad, presentes en las mentes de los estudiantes. A pesar de las dificultades del proyecto, pudo terminarse y exponerse de manera exitosa el producto final: la transmisión del programa de radio en los distintos medios comunicativos, Facebook live y YouTube. Esta herramienta de radio escolar puede replicarse, siguiendo la misma estructura presentada anteriormente, aunque sería recomendable adaptarla al contexto escolar y comunitario específico.

\section{REFERENCIAS}

Abreu, J. (2014). El método de la investigación Research method. International journal of good conscience, 9, 2-9.

Balcázar, F. (2003). Investigación acción participativa (IAP): aspectos conceptuales y dificultades de implementación. Fundamentos en humanidades, 7, 59-77.

Ballesteros López, T. y Martínez Matías, G. (2019). ;La radio vive! Mutaciones culturales de lo sonoro. Colección: estudios culturales y teoría de la medición. Ediciones CIESPAL, 10, 17-53.

Barragán, R. A., Huertas, O., Vargas, V., Cifuentes, F., Castellanos, S., Moreno, C., Castillo, C. y Pardo, O. (2013). La radio escolar y el desarrollo de competencias comunicativas. En Bejarano, L. G. (Eds.). Experiencias de la formación en investigación de los maestros de Cundinamarca (pp. 60-63). Centro Editorial Uniminuto.

Blanco, E., Gómez, B. y Paniagua, F. J. (2014). La utilización de la radio como herramienta didáctica. Una propuesta de aplicación, 39-42.

Cáceres, N. (2020). Educación virtual: creando espacios afectivos de convivencia y aprendizaje en tiempos de COVID-19. CienciAmérica, 9(2), 3-6.

Caldera, I. (2013). La radio escolar: un medio para fortalecer la argumentación oral en estudiantes del grado sexto de la Institución Educativa Simón Bolivar de Planeta Rica Córdoba. Universidad de Antioquia, 16-28.

Campos, S. (2014). Inventando nuevos modos de hacer radio: recuperando el espacio radiofónico desde las participaciones propias en las escuelas Pillao Matao y Huacarpay. Universidad Nacional Mayor de San Marcos, 164.

Catalán, M. (2015). La radio escolar digital y su aporte en la asignatura de Lenguaje y Comunicación en el Colegio Altazor (pp. 103-107). Pontificia Universidad Católica de Chile.

Castilla, B., Calderón, G. y Rojano, J. (2007). La utilización de la radio como herramienta didáctica. Una propuesta de aplicación. FISEC-Estrategias-Facultad de Ciencias Sociales de la Universidad Nacional de Lomas de Zamora, 6, 35-50. 
CONAIE (2014). Chibuleo. Confederación de las Nacionalidades Indígenas del Ecuador. https:// conaie.org/2014/07/19/chibuleo/

Correa, L. y López, A. (2011). La radio escolar como una estrategia de enseñanza-aprendizaje en el colegio Hernando Vélez Marulanda (pp. 22-31). Universidad Tecnológica de Pereira.

Cuevas, M. y Milán, M. L. (2015). Diseño de proyectos escolares. Instituto de formación docente, 12.

Domínguez, R. (2010). Los medios en el aula: el periodismo como recurso pedagógico. Una concienciación necesaria. I Jornadas Universitarias de Comunicación y personas con discapacidad.

Espino, C. y Martín, D. (2012). Las radios universitarias, más allá de la radio. Las TIC como recursos de interacción radiofónica (pp. 21-34). En C. Espino y D. Martín (coords.). Las radios universitarias, más allá de la radio. Las TIC como recursos de interacción radiofónica. UOC.

Figueroa, M. (2006). Cómo crear el departamento de educación: prensa, radio, televisión e internet escolar. Cooperativa editorial magisterio.

Fundación ERPE, (2018). Nuestra historia. https://www.erpe.org.ec/index.php/nosotros/historia

Gallo, L. (2006). El pensamiento educativo de John Locke y la atención a la Educación Física. Universidad de Antioquia.

Guerrero, M. T., Peñuela, M. y Tascón, M. (2012). Los departamentos de comunicación escolar como espacios que desarrollan y potencian habilidades para la vida: el caso del Colegio Lacordaire de Cali-Colombia. Historia de la Educación Colombiana, 15, 323-342.

Kaplún, M. (1999). Producción de programas de radio: el guión-la realización. Ediciones CIESPAL. Colección INTIYÁN. Editorial Quipus.

Martí, J. A., Heydrich, M., Rojas, M. y Hernández, A. (2010). Aprendizaje basado en proyectos: una experiencia de innovación docente. Revista universitaria EAFIT, 46(158), 11-21.

Melgarejo, I. y Rodríguez, M. (2013). La radio como recurso didáctico en el aula de infantil y primaria: los podcasts y su naturaleza educativa. Universidad Católica San Antonio de Murcia, 40-45.

Ministerio de Educación del Ecuador (2017). Currículo Kichwa. Quito-Ecuador.

Ministerio de Educación del Ecuador (2017). Lineamientos pedagógicos para la implementación del Modelo del Sistema de Educación Intercultural Bilingüie. Quito-Ecuador. Acuerdo Ministerial No. 440-13, 68-81.

Ministerio de Educación del Ecuador (2013). MOSEIB: Modelo del Sistema de Educación Intercultural Bilingüe. Quito-Ecuador, 15-17.

Ministerio de Educación del Ecuador (s. f.). Proyecto Educa Televisión para Aprender. https:/ / educacion.gob.ec/ministerio-de-educacion-presento-el-proyecto-educa-television-para-aprender/ ?Fbclid=iwar2pjmrqetntf1lsxmervhsftuuh4aqy7ksyilcngvasnpvqkxuworu-qqu\#: :text=televisi\% C3\%b3n\%20para\%20aprender-,Ministerio $\% 20 \mathrm{de} \% 20 \mathrm{Educaci} \% \mathrm{C} 3 \% \mathrm{~b} 3 \mathrm{n} \% 20$ present $\% \mathrm{C} 3 \% \mathrm{~B} 3 \% 20 \mathrm{el} \% 20$ proyecto $\% 20$ Educa $\% 2 \mathrm{C} \% 20$ televisi\%C3\%b3n\%20para\%20aprender, todas $\% 201$ as $\% 20 \% \mathrm{C} 3 \%$ a1reas $\% 20 \mathrm{del} \% 20$ conocimiento 
Ministerio de Educación del Ecuador (s. f.). Educa Televisión para Aprender estrena nuevos programas. https:/ / educacion.gob.ec/educa-television-para-aprender-estrena-nuevos-programas/?Fbclid=iwar3fdgb2ndbjhw14n4oz-3y64s4cyevu092cajf2i3iszxxvprcuc1o9rqe

Moreno, C. (2015). Posibilidades de la radio escolar como medio para dinamizar el currículo. Opción, 31(2).

Narváez, C. y Pena, D. (2012). Las radios universitarias, más allá de la radio: Las TIC como recurso de interacción radiofónica. Editorial UOC. https://books.google. es / books?hl=es\&lr=\&id=PrDY922FzpkC\&oi=fnd\&pg $=$ PA13\&dq $=\mathrm{LA}+\mathrm{RA}-$ $\mathrm{DIO}+\mathrm{Y}+\mathrm{LAS}+\mathrm{TICS} \& \mathrm{t}_{\mathrm{S}}=1-\mathrm{MJ7acDK1 \& sig=IHE0yyDBcnNRQY9GcwmxwaJhI-}$ $\underline{\mathrm{h} 4 \# \mathrm{v}=\text { onepage } \& \mathrm{q} \& \mathrm{f}=\text { false }}$

Padilla, C. (2015). La radio como estrategia didáctica integradora en el grado décimo de la institución educativa San Simón. Universidad de Tolima, 17-27.

Pérez, A. (2000). Identidad, sentido y uso de la radio educativa, 5-7.

Pérez, J. (2018). La radio escolar en el proceso de enseñanza aprendizaje: el caso de la Escuela de Enseñanza Técnica Provincial $N^{\circ} 459$ de la Ciudad Pérez, Provincia de Santa Fe. Licenciatura en Comunicación Social.

Ramos, C. (2015). Los paradigmas de la investigación científica. file:/ / C: / Users/LENOVO/ Downloads/document\%20(1).pdf

Realinfluencers (2018.) 8 razones para integrar la radio y la televisión en la educación. https:// www.realinfluencers.es/2016/04/11/8-razones-integrar-radio-television-educacion/?Fbclid=iwar0xixzrfnczmciyovriynjxp0kj9wczq07pur_otz72rknkyvj2fkanwha

Rodríguez, I. y Vílchez, J. (2015). El aprendizaje basado en proyectos: un constante desafío. Innovación educativa, 25, 219-234.

Salinas Ibáñez, J. (2008). Innovación educativa y uso de las TIC. Universidad Internacional de Andalucía.

Sánchez, J. (s. f.) Estudios aprendizaje basados en proyectos. Biblioteca CEMLAD.

Secretaría Nacional de Planificación y Desarrollo. Semplades. Plan Nacional de Desarrollo (2017-2021). Toda una vida. Quito-Ecuador.

Subsecretaría de Educación Intercultural Bilingüe (s. f.). Cartilla de saberes y conocimientos de la nacionalidad kichwa pueblo Chibuleo. https:/ / www.educacionbilingue.gob.ec/ wp-content/ uploads/2021/01/CARTILLA-PUEBLO-CHIBULEO-ZONA-3 compressed.pdf

Torres, J. (1994). Globalización e interdisciplinariedad: el currículo integrado (No. 375 T3).

Valderrama, M. y Reyes, B. (2015). ¿Cómo generar espacios de formación radial que fortalezcan las habilidades comunicativas en los estudiantes de noveno y décimo año del Colegio Santa Teresita del Niño Jesús de Villavicencio, meta?, 33-41.

\section{ANEXO}

Enlace del producto final de la propuesta:

https:/ / drive.google.com/file/d/1X7I0Rplzc6qBSfDuBUv27yfsHruwM0Y0/view?us$\mathrm{p}=$ sharing 